\title{
LAS EXPECTATIVAS RACIONALES Y SUS EFECTOS EN LAS VARIABLES REALES DE LA ECONOMÍA
}

\section{Ulises Hernández Ramos*}

\author{
Facultad de Economía \\ Universidad Nacional Autónoma de México
}

(Recibido 1 de noviembre 2004, aceptado 2 de diciembre 2004)

\section{Resumen}

El presente artículo muestra la evolución de las expectativas adaptativas y racionales en la especificación de los modelos macroeconómicos. Se demuestra a través de la especificación formal de los modelos de Lucas y Sargent-Wallace que las políticas no tienen efectos reales en la economía, sin embargo este resultado no se debe a la incorporación de la hipótesis de expectativas racionales, sino al supuesto de vaciado de los mercados, tal y como se demuestra con el modelo formal de Fisher, en el cual, aun considerando expectativas, las políticas sistemáticas tienen efectos en las variables reales de la economía aun en el largo plazo. Se concluye que las expectativas no son el punto central de las controversias, sino de los supuestos sobre los que se construyen los modelos, lo que conduce a las diferencias en los resultados de las políticas contracíclicas del gobierno.

\section{Abstract}

The present article shows the evolution of rational and adaptive expectations in the specification of macroeconomic models. It is demonstrated through the formal specification of the Lucas and Sargent-Wallace models that policies do not have real effects in the economy nevertheless, this result does not obey to the incorporation of the rational expectations hypothesis, but to the drained assumption of the markets, as it is demonstrated with the formal model of Fisher, in which, even considering expectations, the systematic policies even have effects in the real variables of the economy in the long term. One concludes that the expectations are not the central point of the controversies, but of the assumptions on which they are constructed, which leads the differences in the results of the contra cyclic government's policies.

\section{Clasificación JEL: B41, E60, E61}

Palabras clave: Expectativas adaptativas, Expectativas racionales, Instrumentación de políticas, Curva de Phillips

* División de Estudios de Posgrado. Facultad de Economía. Universidad Nacional Autónoma de México. Circuito interior, ciudad universitaria, edificio B, $3^{\circ}$ piso, cubículo 12 . D.F., c.p. 04510. Correo electrónico: uliseshr@economia.unam.mx; uliseshr2000@yahoo.com.mx

Agradezco los comentarios tan valiosos de Javier Galán, Ilana Méndez y David Fonseca. 


\section{Introducción}

Durante la década de los setenta, el consenso sobre la elaboración de políticas basada en el modelo IS-LM de J. Hicks (1937) se enfrentó ante un dilema de carácter teórico y empírico. Desde el punto de vista empírico, el dilema consistía en que el consenso no tenía la capacidad de explicar las elevadas tasas de inflación y desempleo. Mientras que el dilema desde el aspecto teórico era que el consenso dejaba un espacio sin cubrir entre los principios y la práctica macroeconómica bastante grande para ser satisfecha intelectualmente.

Como el modelo IS-LM tradicional tomaba como dado el nivel de precios, se incorporó una curva de Phillips ${ }^{1}$ para explicar el ajuste de precios. Algunos autores pensaban que la curva de Phillips tenía la propiedad de la tasa natural, lo que implicaba que a largo plazo la economía se corregiría por sí sola. Contrario a ello, a finales de la década de los sesenta, los trabajos de Friedman (1968) y Phelps (1968) establecieron que partiendo de principios microeconómicos, esta relación empírica entre inflación y desempleo se rompería si los responsables de las políticas trataban de explotarla, es decir, la curva de Phillips se vio ampliada por un elemento de expectativas, modificando con ello la pendiente de esta curva, pasando desde la perspectiva de corto a largo plazo, hacia la de esperado o no esperado (racionalmente).

Sus argumentos iniciales se basaban en que la tasa de desempleo de equilibrio o natural debía de depender de la oferta y demanda de trabajo, de los tiempos óptimos de búsqueda y de otras consideraciones macroeconómicas, y no de la tasa promedio de crecimiento del dinero.

Tanto el rompimiento del consenso de la curva de Phillips tradicional y las conclusiones de los trabajos de Friedman y Phelps, constituyeron el cimiento para los trabajos posteriores de Lucas (1973) y Sargent-Wallace (1975). A través de estos modelos se mostró que muchas relaciones empíricas que constituían los modelos macroeconómicos de gran escala no se fundamentaban más en los principios microeconómicos que la curva de Phillips. En particular, las decisiones que determinan la mayor parte de las variables macroeconómicas tales como el consumo y la inversión dependían en gran medida de las expectativas que tenían los agentes económicos sobre el futuro comportamiento de la economía.

A través de sus modelos, Lucas mostró que muchas intervenciones de política cambiaban la forma en que los individuos formaban sus expectativas sobre el futuro, la "crítica de Lucas" se convirtió de esta forma en el argumento principal de los radicales que pretendían destruir el consenso. Sin embargo, hoy se sabe que la hipótesis de las expectativas racionales no son el punto de debate, sino los supuestos sobre los que descansan los modelos macroeconómicos.

En 1977 S. Fisher mostró que, siguiendo la misma lógica de Lucas y

1 La curva de Phillips nació a través de una observación puramente empírica de Irving Fisher en 1926, la inflación parecía estar asociada con bajos niveles de desempleo y la deflación con altos niveles. Sin embargo, A. W. Phillips en 1958 reconoció esta misma observación empírica en Inglaterra para el periodo 1861-1957, pero partió su análisis de considerar a la tasa de variación de salarios (vinculada con los precios) como la variable dependiente. Véase Birchenall (1999). 
Sargent-Wallace, ${ }^{2}$ es posible construir modelos con expectativas racionales en los que la política monetaria sistemática puede estabilizar la economía. La única diferencia con el modelo de Lucas y Sargent-Wallace es la incorporación del supuesto de rigideces en el mercado de trabajo mediante contratos laborales, en los que los salarios permanecen rígidos por un tiempo, provocando que las políticas sistemáticas tengan efectos sobre las variables reales en la economía.

A partir del debate anterior, el trabajo se enfoca a analizar estas dos vertientes de investigación argumentando que la hipótesis de expectativas racionales no son el punto de discusión entre las diferencias de las conclusiones en los modelos macroeconómicos, sino en los supuestos sobre los que descansan dichos modelos.

En el segundo apartado se hace un análisis sobre los inicios en la incorporación de las expectativas en el análisis económico, estudiando las expectativas adaptativas de Muth (1961); a través de este estudio, demostramos que mediante la incorporación de estas expectativas se da un gran avance en el diseño de los modelos microeconómicos, pero subsisten deficiencias a partir de que el comportamiento de los agentes es recursivo, construyendo sus expectativas en base a la información de los períodos anteriores y no de la información actual, por lo que no se tiene una reacción inmediata ante los cambios de política.

En el tercer apartado se realiza un análisis de las expectativas racionales y su mecanismo de formulación bajo un esquema de expectativas adaptativas. En el cuarto y quinto apartado se presentan los modelos formales de Lucas y Sargent-Wallace, así como la conclusión principal sobre la irrelevancia de las políticas, manteniendo el supuesto del vaciado de los mercados.

En el sexto apartado se presenta el modelo formal de S. Fisher y su conclusión de la relevancia de las políticas en las variables reales, aun cuando se mantiene el supuesto de expectativas racionales, basando su estudio en las rigideces del mercado de trabajo.

Finalmente, se presentan las conclusiones del trabajo, resaltando la importancia de la hipótesis de las expectativas racionales en el desarrollo de los modelos macroeconómicos para la elaboración de políticas.

\section{Expectativas adaptativas}

Uno de los primeros intentos de establecer un modelo para la revisión sistemática de expectativas a la luz de nueva información fue la hipótesis de las expectativas adaptables introducida por primera vez por Cagan (1956) y Nerlove (1958); hipótesis que fue aceptada en gran medida por la macroeconomía teórica y empírica durante aproximadamente dos décadas.

Esta hipótesis establecía que, dado que los individuos forman sus expectativas en un entorno incierto, es necesario establecer un modelo que logre ejemplificar dicho comportamiento bajo un esquema de elección. Por ejemplo, considerando un individuo que tiene que tomar una elección frente a dos

2 Tanto el modelo de Lucas como el modelo de Sargent-Wallace llegan a la conclusión sobre la irrelevancia de las políticas sistemáticas, ya que sólo pueden generar inflación esperada y, por lo tanto, no pueden producir efectos sobre el producto y el empleo. 
posturas alternativas, ${ }^{3}$ cada una con la misma media pero con diferentes dispersiones o grados de riesgo de desviarse del valor medio, si los individuos afirman que se encuentran indiferentes entre las dos posturas, se dice que son neutrales al riesgo, es decir, que sus preferencias se pueden describir por completo en términos de la media o esperanza matemática del evento incierto; si por el contrario, para dos posturas que tienen la misma media los individuos prefieren la postura que presenta la menor dispersión, entonces se dice que los individuos muestran aversión al riesgo.

Puesto que los individuos valoran los eventos inciertos tanto por la media como por la dispersión, será necesario obtener el modelo de cada una de estas influencias en el comportamiento del individuo. Dado lo anterior, se puede establecer que los individuos valoran subjetivamente las probabilidades y las utilizan para construir el promedio ponderado pertinente en la manera analizada antes.

La formación de las expectativas endógenas es equivalente al establecimiento de una regla mediante la cual los individuos revisan sus expectativas a la luz de información nueva. Basándonos en el trabajo de Cagan (1956), la hipótesis de las expectativas adaptables establece que los individuos utilizan información sobre errores pasados de predicción para revisar sus expectativas actuales.

Si definimos a ${ }_{t-1} x_{t}^{e}$ como el valor en el tiempo $t$ de la variable $x$ que esperan los individuos que forman expectativas al final del tiempo $t-1$, entonces la hipótesis de las expectativas adaptables se establece como:

$$
{ }_{t-1} x_{t}^{e}{ }_{t-2} x_{t-1}^{e}=\phi\left(x_{t-1}-{ }_{t-2} x_{t-1}^{e}\right) \quad 0<\phi<1 .
$$

Cuando se tiene cierta predicción en ${ }_{t-2} x_{t-1}^{e}$ basada en información disponible al final del tiempo $t-2$, los individuos examinan ex post con qué grado de eficacia predice este pronóstico el valor real $x_{t-1}$ y revisan su predicción para $x$ un período después en el tiempo $t$ mediante cierta fracción del error pronosticado en el tiempo $t-1$.

Ordenando nuevamente la primera ecuación, y considerando esta nueva información, nos queda:

$$
{ }_{t-1} x_{t}^{e}=\phi x_{t-1}+(1-\phi)_{t-2} x_{t-1}^{e} .
$$

Bajo el supuesto de que la misma fórmula se mantiene para un período adicional:

$$
{ }_{t-2} x_{t-1}^{e}=\phi x_{t-2}+(1-\phi)_{t-3} x_{t-2}^{e}
$$

Sustituyendo de forma recursiva las expectativas no observables en la segunda ecuación, se obtiene a largo plazo:

$$
{ }_{t-1} x_{t}^{e}=\phi x_{t-1}+\phi(1-\phi) x_{i-2}+\phi(1-\phi)^{2} x_{t-3}+\cdots+\phi(1-\phi)^{n} x_{t-n-1}+(1-\phi)_{t-n-2}^{n+1} x_{t-n-1}^{e} .
$$

3 El caso convencional en la teoría de la probabilidad es el de lanzamiento de una moneda, por lo que las dos alternativas de resultados son conocidas a priori, así como su distribución de probabilidad. 
Esta ecuación indica que todos los términos, excepto el final, son observables. Puesto que $\phi$ es una fracción positiva, $(1-\phi)^{n+1}$ se vuelve cada vez mas pequeña conforme $n$ crece. Con la única condición de que sea infinito el valor de esta expectativa final, se puede reducir su influencia en las expectativas actuales a un nivel infinitesimal si se considera un valor de $n$ suficientemente grande.

El atractivo de la hipótesis de las expectativas adaptables es que nos permite crear un modelo de las expectativas no observables exclusivamente en términos de observaciones pasadas de la variable pertinente $x$, sin necesidad de especificar el proceso con el que se determinó el nivel inicial de las expectativas.

La última ecuación también resalta la regla de comportamiento de la primera ecuación, la cual es en realidad una afirmación de que las expectativas actuales ${ }_{t-1} x_{t}^{e}$ se basan en una extrapolación o promedio ponderado de valores reales pasados de $x$ como se había indicado en el esquema de elección del individuo al inicio del apartado, donde los pesos tienen la propiedad simple de disminución geométrica: el coeficiente de $x_{t-k}$ en la ecuación (3) es $(1-\phi)$ veces el coeficiente de $x_{t-(k-1)}$. Por lo tanto, la regla de las expectativas adaptables se conoce comúnmente como el retardo distribuido geométricamente de los valores pasados de $x$.

La introducción de las expectativas adaptables al análisis económico representaro un avance considerable en el modelaje del comportamiento de los individuos bajo un esquema de incertidumbre; sin embargo, subsistieron varios problemas al interior de estas especificaciones, que posteriormente representaron los cimientos para la incorporación del concepto de las expectativas racionales en los modelos macroeconómicos. Dichas deficiencias fueron:

- La hipótesis de las expectativas adaptables es por completo una visión retrospectiva, como resultado de la ecuación (3). A partir de que sus decisiones se basan en la información pasada, la hipótesis implica que, por ejemplo, la tasa de inflación esperada para el siguiente período es el promedio geométrico ponderado de las tasas de inflación observadas en el presente y las del pasado; sólo después de que la inflación más alta se haya introducido gradualmente en los datos pasados, los individuos simplemente la extrapolarán.

- No se proporciona ninguna teoría económica para explicar la magnitud del parámetro de ajuste $\phi$ en la primera y tercera ecuación.

- La hipótesis considera que las únicas variables que se deben de considerar son valores pasados de la variable acerca de la cual se forman las expectativas.

\section{Expectativas racionales}

En 1961 John Muth formuló una serie de críticas a las expectativas adaptativas y planteó una nueva hipótesis de formación de expectativas según las cuales los agentes usan toda la información relevante disponible, no desperdician información, saben que equivocarse sistemáticamente es costoso por lo que sus predicciones si bien no son exactas deberían ser acertadas en promedio y sus errores deben ser mínimos y aleatorios. Se supone además que los agentes actúan como si entendieran de economía, es decir, como si conocieran el modelo económico relevante. A este tipo de formulación de expectativas, Muth le dio el nombre de expectativas racionales. 
El supuesto fundamental de las expectativas racionales es que los individuos no deberían cometer errores sistemáticos; esto no significa que los individuos predigan invariablemente con exactitud en un mundo donde algunos movimientos aleatorios son inevitables, por el contrario, se afirma que las conjeturas acerca del futuro deben ser correctas en promedio, si los individuos han de quedar satisfechos con su mecanismo de formación de expectativas.

A través de una serie de ejercicios algebraicos, Muth estableció que los agentes optimizadores no se equivocan sistemáticamente, es decir, que las predicciones de los agentes económicos (distribución subjetiva de probabilidades) tienden a coincidir con las predicciones de la teoría económica (distribución objetiva).

Sin embargo, Lucas (1972) establece que el comportamiento racional de los agentes expresado en el no desperdicio de información y la ausencia en los errores sistemáticos, es poco útil para efectos de elaborar teorías. Para Lucas, la hipótesis de expectativas racionales representa una condición de consistencia de los "juegos" que configuran un determinado modelo. De esta forma, una vez que el "juego" es definido, por una cierta función objetivo y un determinado conjunto de restricciones, los agentes establecerán su estrategia óptima para variables de decisión.

Lo anterior implica que las expectativas racionales son equivalentes a decir que los agentes buscan maximizar una cierta función objetivo. El problema conceptual radica, entonces en formalizar este juego, en donde el cambio en las reglas del mismo lleva a modificar la estrategia óptima de los agentes.

Lo anterior implica que las expectativas racionales aseguran que las expectativas subjetivas no observables de los individuos son exactamente las verdaderas esperanzas condicionales matemáticas que implica el modelo mismo, de aquí que los individuos actúan como si conocieran el modelo y crearan expectativas de acuerdo con él.

Utilizaremos una forma esquemática para la modelización de las expectativas racionales a partir de los principios de las expectativas adaptativas, ${ }^{4}$ ya que interesa examinar bajo qué condiciones un esquema de formación de expectativas adaptativas podría ser "racional", lo que hace necesario establecer un tipo de restricción sobre el proceso estocástico de la serie a estimar para que ello ocurra.

Primero consideramos una variable $x$ cuyo proceso estocástico viene dado por la siguiente ecuación:

$$
x_{t}=\varepsilon_{t}+\sum_{i=1}^{\infty} \omega_{i} \varepsilon_{t-i}, \quad \varepsilon \sim \mathcal{N}\left(0, \sigma^{2}\right) .
$$

4 En este ejemplo se considera el comportamiento de una variable en función de su comportamiento histórico, es decir, se consideran las variables que dependen de expectativas endógenas corrientes, se deja ausente la influencia de otras variables externas, ya que se mantiene el supuesto de que esta variable contiene toda la información relevante para la proyección de su nivel futuro. Se dejan al margen otros mecanismos para la especificación de expectativas racionales, como los métodos del operador de adelanto, sustitución repetida o el de coeficientes indeterminados, en los que intervienen expectativas de variables endógenas futuras. Véase Rosende (2002). 
Para este caso, la solución a la ecuación con expectativas adaptativas sería

$$
x_{t}^{e}=\sum_{i=1}^{\infty} \omega_{i} \varepsilon_{t-i} .
$$

Sin embargo, dado que la variable " $\varepsilon$ " no es observable, ésta no puede ser una solución, la solución a este ejercicio consiste en resolver los valores de los coeficientes " $\nu$ ' $s$ " en una ecuación como:

$$
x_{t}^{e}=\sum_{j=1}^{\infty} \nu_{j} x_{t-j} .
$$

De tal forma que éstos sean "racionales", en el sentido de reflejar el comportamiento verdadero de la serie. Reemplazando la ecuación (4) en la ecuación (6), y resolviendo para $x_{t}^{e}$, nos queda:

$$
\begin{gathered}
x_{t}^{e}=\sum_{j=1}^{\infty} \nu_{j}\left[\varepsilon_{t-j}+\sum_{i=1}^{\infty} w_{i} \varepsilon_{t-i-j}\right] \\
x_{t}^{e}=\nu_{1}\left[\varepsilon_{t-1}+\sum_{i=1}^{\infty} w_{i} \varepsilon_{t-i-1}\right]+\nu_{2}\left[\varepsilon_{t-2}+\sum_{i=1}^{\infty} w_{i} \varepsilon_{t-i-2}\right]+\cdots
\end{gathered}
$$

La ecuación (7) puede escribirse como:

$$
x_{t}^{e}=\nu_{1} \varepsilon_{t-1}+\sum_{i=2}^{\infty} \varepsilon_{t-1}\left[\nu_{i}+\sum_{j=1}^{i-1} \nu_{j} w_{i-j}\right] .
$$

Lo que permite establecer una identidad entre los coeficientes de las ecuaciones (5) y (8). De aquí se obtiene que:

$$
\begin{aligned}
& \omega_{1}=\nu_{1}, \\
& \omega_{2}=\nu_{2}+\sum_{j=1}^{2-1} \nu_{j} w_{2-j}=\nu_{2}+\nu_{1} w_{1}
\end{aligned}
$$

y de forma general se cumple

$$
\mathrm{w}_{i}=\nu_{i}+\sum_{j=1}^{i-1} \nu_{j} w_{i-j} .
$$

El siguiente paso es establecer bajo que condiciones de la serie en cuestión un esquema de expectativas adaptativas sería "racional". En este caso la interrogante se dirige hacia la forma de la estructura de ponderaciones de los coeficientes " $\omega$ ' $s$ ", considerando que la utilización de un modelo de expectativas adaptativas supone una estructura de ponderaciones declinante como:

$$
x_{t}^{e}=\beta \sum_{i=0}^{\infty}(1-\beta)^{i} x_{t-i-1}=\beta \sum_{i=1}^{\infty}(1-\beta)^{i-1} x_{t-i}, \quad 0<\beta<1 .
$$


Dado que en las ecuaciones (6) y (11) se pueden igualar los coeficientes, tal que para todo " $i$ " se cumple que $\nu_{i}=\beta(1-\beta)^{i-1}$. Utilizando la información de la ecuación (9), se obtiene:

$$
\begin{aligned}
\omega_{1} & =\nu_{1}=\beta, \\
\omega_{2} & =\nu_{2}+\nu_{1} \omega_{1}=\beta(1-\beta)+\beta^{2}=\beta, \\
\omega_{3} & =\nu_{3}+\sum_{j=1}^{2} \nu_{j} \omega_{3-j}=\nu_{3}+\nu_{1} \omega_{2}+\nu_{2} \omega_{1} \\
& =\beta(1-\beta)^{2}+\beta \beta+\beta^{2}(1-\beta)=\beta .
\end{aligned}
$$

Finalmente, si para todo $\omega_{i}=\beta$, entonces un proceso adaptativo es racional. En este caso, el comportamiento verdadero de la serie $x$ se describe por la ecuación:

$$
x_{t}=\varepsilon_{t}+\beta \sum_{i=1}^{\infty} \varepsilon_{t-i} .
$$

Una de las primeras implicaciones de las expectativas racionales establece que si los agentes económicos forman sus expectativas de manera racional, la política económica sistemática será inefectiva inclusive en el corto plazo. R. Lucas (1972,1973), Sargent y Wallace (1975), Barro y Gordon (1983a,1983b) encabezaron esta nueva corriente que fue denominada como los nuevos clásicos pues llegaban a la misma conclusión de que la política económica no era necesaria para alcanzar el pleno empleo, ya que este se realizaba de forma automática. ${ }^{5}$

El trabajo de Sargent y Wallace establecía que la política monetaria sistemática es irrelevante para la trayectoria de la producción y el empleo. Para llegar a esta conclusión los autores sólo aplicaron las expectativas racionales a la curva de Phillips aumentada con expectativas, utilizada por Friedman (1968) y Phelps (1968). Sargent y Wallace concluyeron que la política monetaria sistemática puede generar solamente inflación esperada, no puede producir inflación no esperada y, por lo tanto, no puede afectar el desempleo.

Contrario a lo anterior, en 1977 S. Fisher publicó un artículo en el que establecía que es completamente posible construir modelos con expectativas racionales en los que la política monetaria sistemática puede estabilizar la economía. Fisher elaboró su modelo bajo el supuesto de la existencia de contratos laborales periódicos, por lo que los salarios inflexibles desempeñarían un papel crucial para la estabilización económica, tal y como lo establecía las

5 Los trabajos de Barro y Gordon (1983a,1983b), Kydland y Prescott (1977), se dirigieron al estudio de la valoración de las políticas publicas en sentido discrecional o bajo reglas determinadas. Los temas de credibilidad en la aplicación de políticas, así como de inconsistencias dinámicas fueron el centro de discusión al margen de la efectividad de la política monetaria sobre las variables reales de la economía. Estos temas no se discuten directamente en el presente trabajo por ser extensiones de nuestro tema, sin embargo un ejemplo del análisis bajo un enfoque de credibilidad y reputación de la política monetaria, para el caso específico del comportamiento de la política monetaria en México se encuentra en Fonseca, D. et al. (2004). 
prescripciones de la política keynesiana. Dado este resultado se llegó a un consenso general de que la irrelevancia de la política no es la implicación de las expectativas racionales per se.

Como una forma de contrastar estas dos visiones de las expectativas racionales y sus implicancias para las políticas económicas, en los dos siguientes apartados se establecen los modelos de Lucas-Sargent-Wallace y el de Fisher.

\section{El Modelo de Lucas}

Tanto el modelo de Lucas, como el de Sargent y Wallace, inician con tres supuestos fundamentales: a) los mercados se vacían, b) existe una tasa natural de desempleo independiente del ciclo y c) las acciones de los agentes económicos son racionales.

En los artículos de Lucas $(1972,1973)$ se establece que existe una relación positiva entre empleo e inflación a corto plazo, implicando que el modelo tiene todas las propiedades del modelo clásico y, por lo tanto, con equilibrio en el mercado de trabajo y en el de bienes. Los agentes económicos confunden el aumento del nivel general de precios, debido a un aumento de la oferta monetaria, con cambios en los precios relativos de los bienes. ${ }^{6}$

Lucas inicia su modelo estableciendo que la economía se encuentra compuesta por $z$ mercados independientes ${ }^{7}$ :

$$
\begin{gathered}
n_{t}^{d}(z)=\frac{\ln (1-\alpha)}{\alpha}+\bar{k}(z)+\frac{1-\alpha}{\alpha} \phi_{t}(z)-\frac{1}{\alpha}\left(w_{t}(z)-p_{t}(z)\right), \\
n_{t}^{s}(z)=\bar{n}(z)+b_{1}\left(w_{t}(z)-\mathrm{E}\left[p_{t} / I_{t}(z)\right]\right), \\
n_{t}^{d}(z)=n_{t}^{s}(z), \\
y_{t}^{s}(z)=\alpha \bar{k}(z)+(1-\alpha) n_{t}^{d}(z)+(1-\alpha) \phi_{t}(z) .
\end{gathered}
$$

El salario de equilibrio en el mercado $z$, viene determinado por:

$w_{t}(z)=\frac{\alpha}{\alpha b_{1}+1}\left(\bar{k}(z)+\frac{1-\alpha}{\alpha} \phi(z)+\frac{\ln (1-\alpha)}{\alpha}-\bar{n}(z)+\frac{1}{\alpha} p_{t}(z)+b_{1} \mathrm{E}\left[p_{t} / I_{t}(z)\right]\right)$.

6 En el modelo, Lucas supone que frente a alteraciones en el patrón de demanda que enfrenta cada sector de la economía, los agentes confunden los movimientos de la demanda agregada con cambios en la demanda sectorial. Sin embargo, en el largo plazo y manteniendo el supuesto de que los agentes son racionales, no existe la posibilidad de desviar variables reales, como el producto y el empleo, de sus niveles "naturales", por cuanto los agentes económicos serán capaces de aislar la influencia de "shocks globales" de los "sectoriales".

7 El supuesto de que la economía se encuentra formada por una serie de "islas" ha sido fuertemente criticado; sin embargo, es muy útil si se considera que en el corto plazo los individuos sólo observan lo que ocurre en su propio sector, de lo cual toman sus decisiones de producción. Sin embargo, para los periodos subsiguientes, los agentes pueden desplazarse a otras "islas" e informarse en forma más precisa de lo que ocurre en el resto de la economía, para separarse de lo que realmente serían los shocks sectoriales y los shocks globales. 
Dado lo anterior, el empleo de equilibrio será:

$$
n_{t}(z)=\mu_{0}+\mu_{1} \bar{k}(z)+\mu_{2} \phi(z)+\frac{b_{1}}{\alpha b_{1}+1}\left(p_{t}(z)-\mathrm{E}\left[p_{t} / I_{t}(z)\right]\right)
$$

y el producto producido en el z-ésimo mercado en el periodo $t$ vendrá dado por:

$$
y_{t}^{s}(z)=\bar{y}(z)+\frac{(1-\alpha) b_{1}}{\alpha b_{1}+1}\left(p_{t}(z)-\mathrm{E}\left[p_{t} / I_{t}(z)\right]\right)+u_{t}^{s}(z) .
$$

Lucas establece que el precio en cada mercado se encuentra expuesto a shocks de carácter específico, esto lo podemos representar como: $p_{t}(z)=p_{t}+\varepsilon_{t}^{d}(z)$.

Ahora considerando las expectativas de los agentes, cuando los individuos calculan las expectativas del nivel general de precios con la información disponible en cada mercado: $I_{t}(z)=\left\{I_{t-1}, p_{t}(z)\right\}$, estos cálculos serán comunes para todos los mercados:

$$
p_{t}-\mathrm{E}\left[p_{t} / I_{t-1}\right]=\nu_{t}
$$

Por lo que el precio en todos los mercados será:

$$
p_{t}(z)=\mathrm{E}\left[p_{t} / I_{t-1}\right]+\nu_{t}+\varepsilon_{t}^{d}
$$

existe ortogonalidad de $\nu_{t}$ y $\varepsilon_{t}^{d} \operatorname{si} \operatorname{var}\left(\nu_{t}+\varepsilon_{t}^{d}\right)=\sigma_{\nu}^{2}+\sigma_{\varepsilon^{d}}^{2}$.

Como $\nu_{t}$ y $\varepsilon_{t}^{d}$ no forman parte del conjunto de información en $t-1$, ello implicará que: $\mathrm{E}\left[p_{t} / I_{t-1}\right]=\mathrm{E}\left[p_{t}(z) / I_{t-1}\right]$. Cuando los agentes económicos conocen el nivel de precios en su mercado revisarán sus expectativas sobre el nivel de precios, estimando la siguiente expresión:

$$
p_{t-j}-\mathrm{E}\left[p_{t-j} / I_{t-j-1}\right]=k\left(p_{t-j}(z)-\mathrm{E}\left[p_{t-j} / I_{t-j-1}\right]\right) .
$$

Como

$$
p_{t-j}-\mathrm{E}\left[p_{t-j} / I_{t-j-1}\right]=\nu_{t-j} \quad \text { y } \quad p_{t-j}(z)-\mathrm{E}\left[p_{t-j} / I_{t-j-1}\right]=\nu_{t-j}+\varepsilon_{t-j}^{d}
$$

Entonces:

$$
k=\frac{\sigma_{\nu}^{2}}{\sigma_{\nu}^{2}+\sigma_{\varepsilon^{d}}^{2}} .
$$

Agrupando términos

$$
\mathrm{E}\left[p_{t} / I_{t}(z)\right]=k p_{t}(z)+(1-k) \mathrm{E}\left[p_{t} / I_{t-1}\right]
$$

Finalmente, la curva de oferta en cada mercado es:

$$
y_{t}^{s}(z)=\bar{y}(z)+\frac{\alpha b_{1}}{\alpha b_{1}+1}(1-k)\left(p_{t}(z)-\mathrm{E}\left[p_{t} / I_{t-1}\right]\right)+u_{t}^{s}(z)
$$

y la curva de oferta agregada de Lucas vendrá dada por

$$
y_{t}^{s}=\bar{y}+\beta\left(p_{t}+\mathrm{E}\left[p_{t} / I_{t-1}\right]\right)+u_{t}^{s}
$$


Esta curva de oferta implica que, la única forma a través de la cual la política del gobierno puede producir desviaciones respecto a la tasa natural de desempleo es induciendo a los agentes privados a tener expectativas equivocadas. La función de oferta agregada implica que las desviaciones de la producción respecto a la tasa natural son directamente proporcionales a las desviaciones de los precios respecto a sus valores esperados. Bajo la hipótesis de expectativas racionales, lo agentes privados no cometen errores sobre las reglas de política, es decir, nunca serán engañados de forma sistemática y, por lo tanto, la producción no se desviará respecto a su tasa natural como resultado de cualquier regla de política.

\section{El Modelo de Sargent-Wallance}

Sargent y Wallace (1975) elaboran un modelo utilizando la curva de Phillips ampliada con expectativas, destacando que la política monetaria sistemática no tiene efectos sobre el empleo y el producto. Los autores inician estableciendo una función de demanda:

$$
\begin{gathered}
y_{t}^{s}=\bar{y}+\beta\left(p_{t}-p_{t} / t-1\right)+u_{t}^{s}, \\
y^{d}=\bar{\nu}+m_{t}-p_{t}, \\
m_{t}=\phi u_{t-1}+\varepsilon_{t}^{m}, \\
y_{t}=y_{t}^{s}=y_{t}^{d} .
\end{gathered}
$$

Como la función de demanda agregada es decreciente con respecto a los precios, se desplazará cuando se incrementa la oferta de dinero. El equilibrio entre oferta y demanda agregada vendrá dada por:

$$
p_{t}=\frac{1}{1+\beta}\left(m_{t}+\bar{\nu}-\bar{y}+\beta p_{t / t-1}-u_{t}^{s}\right) .
$$

Se necesita conocer la sorpresa en precios:

$$
p_{t / t-1}=\frac{1}{1+\beta}\left(m_{t / t-1}+\bar{\nu}-\bar{y}+\beta p_{t / t-1}-u_{t / t-1}^{s}\right),
$$

teniendo en cuenta que $u_{t}^{s}-u_{t / t-1}^{s}=\varepsilon_{t}^{s}$, nos queda:

$$
p_{t}-p_{t / t-1}=\frac{1}{1+\beta}\left(m_{t}-m_{t / t-1}\right)-\frac{1}{1+\beta} \varepsilon_{t}^{s} .
$$

La forma reducida del nivel de producción vendrá dada por:

$$
y_{t}=\bar{y}+\theta\left(m_{t}-m_{t} / t-1\right)+u_{t},
$$

en donde

$$
\theta=\frac{\beta}{1+\beta}>0 \quad y \quad u_{t}=u_{t}^{s}-\frac{\beta}{1+\beta} \varepsilon_{t}^{s}
$$


Una de las primeras implicaciones del modelo de Sargent-Wallace es que sólo el componente no anticipado de la política monetaria $\left(m_{t}-m_{t / t-1}\right)$ puede afectar a $\left(y_{t}-\bar{y}\right) \mathrm{y}$, por lo tanto, la política monetaria anticipada es neutral. Como:

$$
\begin{aligned}
y_{t} & =\bar{y}+\theta \varepsilon_{t}^{m}+u_{t}, \\
\operatorname{var}\left(y_{t}\right) & =\theta^{2} \sigma_{m}^{2}+\sigma_{u}^{2} .
\end{aligned}
$$

La elección del parámetro $\theta$ no influye sobre la varianza del producto.

\section{El Modelo de S. Fisher}

Uno de los primeros intentos por apartarse del paradigma walrasiano en el mercado de trabajo, pero manteniendo el supuesto de expectativas racionales consistió en la utilización de rigideces en los salarios nominales. El modelo de Fisher (1977) constituye una aportación a esta línea de estudio, aun cuando su estructura lógica es muy similar al modelo de Sargent-Wallace, se incorpora el supuesto de que los salarios nominales se negocian con antelación a la determinación de la política monetaria anticipada, es decir, mantiene el supuesto de contratos laborales ex ante, por lo que cualquier perturbación posterior a la que puede ser sometida la economía ocasiona un desequilibrio en el mercado de trabajo.

A partir del modelo de Fisher se demostró que la proposición de irrelevancia de la política monetaria anticipada que aparecía en el modelo de SargentWallace no se debía al supuesto de expectativas racionales, sino al de equilibrio walrasiano de los mercados.

En este modelo se abandona el supuesto de vaciado de los mercados por el de rigideces en los salarios; ante desequilibrios en el mercado de trabajo, la política monetaria puede afectar al producto a corto plazo a pesar de que las expectativas sean racionales, por tanto, existe un justificativo para el uso de políticas de estabilización.

Iniciamos el modelo de Fisher considerando nuevamente la curva de oferta de Lucas:

$$
y_{t}^{s}=\bar{y}+\beta\left(p_{t}-p_{t / t-1}\right)+u_{t}^{s}, \quad \beta>0 .
$$

Como habíamos establecido antes mediante esta ecuación, la única manera que tiene la autoridad monetaria de conseguir que el producto actual se sitúe por encima de su tasa natural es mediante una diferencia entre el nivel de precios y su expectativa. Fisher introduce la existencia de contratos laborales en la economía que mantienen constantes las variables nominales durante cierto tiempo, además también se mantiene el supuesto de la ausencia de problemas de información entre los mercados, por lo que:

$$
\begin{gathered}
n_{t}^{d}=\frac{\ln (1-\alpha)}{\alpha}+\bar{k}+\frac{1-\alpha}{\alpha} \phi_{t}-\frac{1}{\alpha}\left(w_{t}-p_{t}\right), \\
n_{t}^{s}=\bar{n}+b_{1}\left(w_{t}-p_{t}\right), \\
y_{t}=\alpha k_{t}+(1-\alpha) n_{t}+(1-\alpha) \phi_{t}, \\
w_{t}=w_{t}^{t-j}=w_{t / t-j}^{*}, \\
n_{t}=n_{t}^{d},
\end{gathered}
$$


Considerando el salario negociado como:

$$
w_{t / t-j}^{*}=\left\{W^{*} / n_{t / t-j}^{d}\left(W^{*}\right)=n_{t / t-j}^{s}\left(W^{*}\right)\right\} .
$$

El salario de equilibrio en $t$ vendrá dado cuando: $n_{t}^{d}=n_{t}^{s}$, por lo que la curva de oferta vendrá dada por:

$$
\mathrm{w}_{t}^{*}=p_{t}+\frac{\alpha}{\alpha b_{1}+1}\left(\frac{\ln (1-\alpha)}{\alpha}+\bar{k}+\frac{1-\alpha}{\alpha} \phi_{t}-\bar{n}\right) .
$$

El salario negociado es el valor esperado en $t-j$ :

$$
w_{t / t-j}^{*}=p_{t / t-j}+\frac{\alpha}{\alpha b_{1}+1}\left(\frac{\ln (1-\alpha)}{\alpha}+\bar{k}-\bar{n}\right)
$$

y el empleo vendrá dado por la demanda de trabajo a ese nivel de salario

$$
n_{t}=\frac{\alpha b_{1}}{\alpha b_{1}+1}\left(\frac{\ln (1-\alpha)}{\alpha}+\vec{k}+\frac{1-\alpha}{\alpha} \phi_{t}\right)+\frac{1}{\alpha b_{1}+1} \bar{n}+\frac{1}{\alpha}\left(p_{t}-p_{t / t-j}\right) .
$$

Esta ecuación indica que si la economía no se encuentra sometida a ningún shock de oferta, el salario negociado en $t-j$ coincidirá con el de equilibrio. Si se produce un shock de oferta negativo que no fuese previsto por los agentes económicos, la demanda de trabajo en $t$, para cualquier nivel de los salarios nominales, será inferior a la demanda de trabajo prevista en $t-j$. La oferta agregada de la economía será entonces:

$$
y_{t}^{s}=\bar{y}+\beta\left(p_{t}-p_{t / t-j}\right)+u_{t}^{s} .
$$

Esta ecuación de oferta agregada incorpora la noción keynesiana de que el salario nominal, lejos de ajustarse instantáneamente para vaciar el mercado de trabajo, se encuentra fijado de antemano.

Ahora pasaremos a analizar dos casos de negociaciones temporales de los contratos laborales:

a) Caso en el que $j=1$,

$$
\begin{aligned}
y_{t}^{s} & =\bar{y}+\beta\left(p_{t}-p_{t / t-1}\right)+u_{t}^{s}, \\
y_{t}^{s} & =\bar{\nu}+m_{t}-p_{t}, \\
y_{t}^{s} & =y_{t}^{d}=y_{t} \\
m_{t} & =\phi u_{t-1}+\varepsilon_{t}^{m} .
\end{aligned}
$$

Este modelo es en apariencia igual al modelo de Sargent-Wallace, ya que mantiene la propiedad de neutralidad; sin embargo, la pendiente de la curva de oferta $(\beta)$ es en este caso independiente de los parámetros de política monetaria.

b) Caso en el que $j=2$,

$$
y_{t}^{s}=\bar{y}+\beta\left(p_{t}-p_{t / t-2}\right)+u_{t}^{s} .
$$


Manteniendo el supuesto de que las perturbaciones de oferta se encuentran autocorrelacionadas: $u_{t}^{s}=\rho u_{t-1}^{s}+\varepsilon_{t}^{s}$, el modelo se resolverá de la forma:

$$
y_{t}=\bar{y}+\phi\left(m_{t}-m_{t / t-2}\right)+u_{t}
$$

donde:

$$
u_{t}=\rho^{2} u_{t-2}^{s}+\frac{1}{1+\beta}\left(\varepsilon_{t}^{s}+\rho \varepsilon_{t-1}^{s}\right)
$$

Esto significa que la política monetaria anticipada en $t-1$ influye en gran medida en la desviación del producto respecto a su tasa natural $\left(y_{t}-\bar{y}\right)$. Lo podemos observar resolviendo el sistema:

$$
\begin{aligned}
m_{t} & =\phi u_{t-1}+\varepsilon_{t}^{m}=\phi \rho^{2} u_{t-3}^{s}+\frac{\phi}{1+\beta}\left(\varepsilon_{t-1}^{s}+\rho \varepsilon_{t-2}^{s}\right)+\varepsilon_{t}^{m}, \\
m_{t / t-2} & =\phi \rho^{2} u_{t-3}^{s}+\frac{\phi}{1+\beta} \rho \varepsilon_{t-2}^{s}, \\
m_{t}-m_{t / t-2} & =\frac{\phi}{1+\beta} \varepsilon_{t-1}^{s}+\varepsilon_{t}^{m} \\
y_{t} & =\bar{y}+\frac{1}{1+\beta}(\theta \phi+\rho) \varepsilon_{t-1}^{s} \theta \varepsilon_{t}^{m}+\rho^{2} u_{t-2}^{s}+\frac{1}{1+\beta} \varepsilon_{t}^{s} .
\end{aligned}
$$

Aun cuando los agentes económicos anticipan en $t-1$ un componente importante de $m_{t}$, no pueden alterar sus contratos nominales para $t$ debido a que fueron negociados en $t-2$. Por lo tanto, si la oferta monetaria se incrementa en $t$, aun siendo un cambio anticipado y, con ella los precios, el salario real disminuye, provocando un incremento en el nivel de empleo y, de ahí, en el producto.

Existe también otro razonamiento intuitivo de los efectos de la política monetaria sobre las variables reales de la economía utilizando la hipótesis de las expectativas racionales, por ejemplo, en el caso en que exista una deficiencia en la demanda agregada en un modelo de tipo keynesiano, el argumento tradicional es que la expansión de la política monetaria para contrarrestar esta deficiencia en la demanda terminará afectando las tasas de interés de tal forma que la economía pasará a un nivel mas alto de producto. Incorporando la hipótesis de expectativas racionales, este paso al nuevo nivel sería bastante rápido, ya que los agentes económicos comprenderían las implicaciones económicas de esta política expansiva del gobierno, de esta forma, estos agentes podrían expandir su producción, en anticipación a los efectos que se esperarían por el aumento en la demanda que tendrían que hacerse obvios en el mercado. Para este caso, lejos de que la política monetaria sea ineficiente como un mecanismo contracíclico, las expectativas racionales pueden incluso hacer que esta política sea más efectiva sobre las variables reales.

\section{Conclusiones}

La incorporación de las expectativas en los modelos macroeconómicos ha revolucionado en gran medida el análisis del comportamiento de los agentes económicos ante cambios de política del gobierno. Los resultados obtenidos mediante la especificación de los modelos han tenido gran significancia debido a los supuestos 
sobre los que se parte, por lo que las connotaciones ideológicas no se encuentran ausentes en estos resultados.

Analizando los modelos de Lucas, Sargent-Wallace y Fisher pudimos observar que el punto neurálgico de los resultados de las políticas contracíclicas no se encuentra en la incorporación o no de la hipótesis de las expectativas racionales, sino del supuesto del vaciado de los mercados y de las rigideces de los salarios. Visto de esta forma, desde el ámbito de la academia, la teoría de las expectativas racionales podrá ser vista como un desarrollo importante en la economía, no por el resultado de ineficiencia de las políticas económicas, sino por su importancia para cualquier situación en la que el comportamiento de los mercados pueda ser influenciado por las expectativas en un entorno de incertidumbre económica.

Los resultados del modelo de Sargent-Wallace condujeron a pensar en la irrelevancia de la política monetaria sistemática para contrarrestar ineficiencias en la demanda agregada, por lo que establecían que la aplicación de esta política generaría solamente inflación esperada y no podrían afectar al producto y al empleo. Además, Lucas argumentó que la única forma de incentivar el producto y el empleo era mediante sorpresas inflacionarias, es decir, mediante engaños a los agentes económicos. Lejos de estas posiciones teóricas, en el ámbito real durante la década de los setenta, los cambios de las autoridades monetarias tenían efectos sustanciales y de largo plazo en la producción y el desempleo, sin importar que los cambios de política monetaria fuesen esperados o no (Cassidy, 1998), resultados que contradecían los principios básicos de estos modelos.

Con el modelo de Fisher, se pudo comprobar que a pesar de que se mantenga la hipótesis de expectativas racionales y, dentro de la lógica de los modelos de Lucas, las rigideces en el mercado de trabajo puede provocar que las políticas monetarias contracíclicas tengan efectos reales en el producto y el empleo; sin embargo, adolece de una falta con la evidencia empírica, ya que en este modelo el salario real es contracíclico cuando la evidencia empírica, en todo caso, parece ser lo contrario.

Lejos de generar mayores diferencias entre los planteamientos de estos autores, debemos establecer que con el desarrollo de la teoría de las expectativas racionales se logrará una mayor comprensión y significancia en la contribución a la economía en el aspecto de especificación de los modelos macroeconómicos, también incluirá importantes cuestiones acerca del uso y la disponibilidad de la información.

\section{Bibliografía}

Barro, R. and D. Gordon (1983a). A Positive Theory of Monetary Policy in a Natural Rate Model. Journal of Political Economy, (91).

Barro, R. and D. Gordon (1983b). Rules, Discretion and Reputation in a Model of Monetary Policy. Journal of Monetary Economics, (12).

Begg, D. (1989). La revolución de las expectativas racionales en la macroeconomía. FCE, México.

Birchenall, A. (1999). La curva de Phillips, la crítica de Lucas y la persistencia de la inflación en Colombia. Monetaria, CEMLA, enero-marzo, pp. 87-125.

Cagan, P. (1956). The Monetary Dynamics of Hyperinflation. In M. Friedman (Ed.). Studies in The Quantity Theory of Money. University of Chicago Press. 
Cassidy, J. (1998). La decadencia de la economía. Economía Informa, 263, pp. 5-13.

Febrero, R. (2000). La moderna macroeconomía clásica y sus consecuencias para la formulación de la política económica. Documento de Trabajo, 2000-1, IUDEM, España.

Fisher, S. (1977), Long-Term Contracts, Rational Expectations, and the Optimal Money Supply Rule. Journal of Political Economy, 85(1), pp. 191-205.

Fonseca, D., J. Galán y U. Hernández (2004). Banco de México: credibilidad y gubernatura. Carta de Políticas Públicas, 6(36).

Friedman, M. (1968). The Role of Monetary Policy. The American Economic Review, 58(1) pp. $1-17$.

Hicks, J. R. (1937). Mr. Keynes and the "Classics": A Suggested Interpretation. Econometrica, 5, pp. 147-159

Hicks, J. R. (1989). IS-LM: una explicación en dinero, interés y salarios. FCE, México.

Keynes, J. M. (2000). Teoría general de la ocupación, el interés y el dinero. FCE, México.

Kydland, F. and E. Prescott (1977). Rules Rather than Discretion: The Inconsistency of Optimal Plans. Journal of Political Economy, 85(3), pp. 473-492.

Lucas, R. (1973). Some International Evidence on Output-Inflation Tradeoffs. American Economic Review, 63(39, pp. 326-334.

Lucas, R. (1972). Expectations and the Neutrality of Money. Journal of Economic Theory, 4, pp. 103-124.

Maddock, R. and M. Carter (1982). A Childs Guide to Rational Expectations. Journal of Economic Literature, XX, pp. 39-51.

Mankiw, G. (1992). Curso rápido sobre Macroeconomía. Investigación Económica, 201, pp. 243-269.

Muth, J. (1961). Rational Expectations and the Theory of Price Movements. Econometrica, 29(3), pp. 315-335.

Nerlove, M. (1958). Adaptive Expectations and Cobweb Phenomena. Quarterly Journal of Economics, 72, pp. 227-240.

Phelps, E. (1968). Money-Wage Dynamics and Labor Market Equilibrium. Journal of Political Economics, 76(4), pp. 687-711.

Romer, D. (2002). Macroeconomía Avanzada. Mc Graw Hill.

Rosende, F. (2002). Teoría Macroeconómica, ciclos económicos, crecimiento e inflación. Alfaomega, Colombia.

Sachs, J. y F. Larraín (1994). Macroeconomía en la economía global. Prentice Hall Hispanoamericana, México.

Sargent, T. and N. Wallace (1975). Rational Expectations, The Optimal Monetary Instrument, and The Optimal Money Supply Rule. Journal of Political Economy, 83(2), pp. $241-254$. 